\title{
Complete and incomplete Bell polynomials associated with Lah-Bell numbers and polynomials
}

\author{
Taekyun Kim', Dae San Kim², Lee-Chae Jang ${ }^{3 *}$, Hyunseok Lee ${ }^{1}$ and Han-Young Kim
}

"Correspondence:

Lcjang@konkuk.ac.kr

${ }^{3}$ Graduate School of Education,

Konkuk University, Seoul 05029

Republic of Korea

Full list of author information is

available at the end of the article

\section{Springer}

\begin{abstract}
The $n$th $r$-extended Lah-Bell number is defined as the number of ways a set with $n+r$ elements can be partitioned into ordered blocks such that $r$ distinguished elements have to be in distinct ordered blocks. The aim of this paper is to introduce incomplete $r$-extended Lah-Bell polynomials and complete $r$-extended Lah-Bell polynomials respectively as multivariate versions of $r$-Lah numbers and the $r$-extended Lah-Bell numbers and to investigate some properties and identities for these polynomials. From these investigations we obtain some expressions for the $r$-Lah numbers and the $r$-extended Lah-Bell numbers as finite sums.
\end{abstract}

MSC: 11B73; 11B83; 05A19

Keywords: Incomplete $r$-extended Lah-Bell polynomial; Complete $r$-extended Lah-Bell polynomial

\section{Introduction}

It is well known that the unsigned Lah number $L(n, k)(n \geq k \geq 0)$ counts the number of ways a set with $n$ elements can be partitioned into $k$ nonempty linearly ordered subsets (see $[4,7,8])$. The $n$th Lah-Bell number $B_{n}^{L}(n \geq 0)$ is the number of ways a set with $n$ elements can be partitioned into nonempty linearly ordered subsets. Thus

$$
B_{n}^{L}=\sum_{k=0}^{n} L(n, k) \quad(n \geq 0)(\text { see }[7,8])
$$

From (1) it follows that the generating function of Lah-Bell numbers is given by

$$
e^{\frac{t}{1-t}}=\sum_{n=0}^{\infty} B_{n}^{L} \frac{t^{n}}{n !} \quad(\text { see }[7,8])
$$

where

$$
\frac{1}{k !}\left(\frac{t}{1-t}\right)^{k}=\sum_{n=k}^{\infty} L(n, k) \frac{t^{n}}{n !} \quad(k \geq 0)(\operatorname{see}[7,13,15,17]) .
$$

(c) The Author(s) 2021. This article is licensed under a Creative Commons Attribution 4.0 International License, which permits use sharing, adaptation, distribution and reproduction in any medium or format, as long as you give appropriate credit to the original author(s) and the source, provide a link to the Creative Commons licence, and indicate if changes were made. The images or other third party material in this article are included in the article's Creative Commons licence, unless indicated otherwise in a credit line to the material. If material is not included in the article's Creative Commons licence and your intended use is not permitted by statutory regulation or exceeds the permitted use, you will need to obtain permission directly from the copyright holder. To view a copy of this licence, visit http://creativecommons.org/licenses/by/4.0/. 
Explicitly, we see from (3) that the Lah numbers are given by

$$
L(n, k)=\frac{n !}{k !}\left(\begin{array}{l}
n-1 \\
k-1
\end{array}\right) \quad(n \geq k \geq 0)(\text { see }[7-10,17,19]) .
$$

Let $n, k, r$ be nonnegative integers with $n \geq k$. Then the $r$-Lah number $L_{r}(n, k)$ counts the number of partitions of a set with $n+r$ elements into $k+r$ ordered blocks such that $r$ distinguished elements have to be in distinct ordered blocks (see [17]). The $r$-extended Lah-Bell number $B_{n, r}^{L}$ is defined as the number of ways a set with $n+r$ elements can be partitioned into ordered blocks such that $r$ distinguished elements have to be in distinct ordered blocks (see [8]). By the definitions of $r$-Lah numbers and $r$-extended Lah-Bell numbers we have

$$
B_{n, r}^{L}=\sum_{k=0}^{n} L_{r}(n, k) \quad(n \geq 0)(\text { see }[8]) .
$$

From (4) we see that the generating function of $r$-extended Lah-Bell numbers is given by

$$
e^{\frac{t}{1-t}}\left(\frac{1}{1-t}\right)^{2 r}=\sum_{n=0}^{\infty} B_{n, r}^{L} \frac{t^{n}}{n !} \quad(\text { see }[8,15])
$$

where

$$
\frac{1}{k !}\left(\frac{t}{1-t}\right)^{k}\left(\frac{1}{1-t}\right)^{2 r}=\sum_{n=k}^{\infty} L_{r}(n, k) \frac{t^{n}}{n !} \quad(\text { see }[8,17])
$$

for nonnegative integers $k$.

Explicitly, the $r$-Lah numbers are given by

$$
L_{r}(n, k)=\frac{n !}{k !}\left(\begin{array}{l}
n+2 r-1 \\
k+2 r-1
\end{array}\right) \quad(n \geq k \geq 0)(\text { see }[7-10,17,19]) .
$$

In [8] the $r$-extended Lah-Bell polynomials are defined by

$$
e^{x\left(\frac{t}{1-t}\right)}\left(\frac{1}{1-t}\right)^{2 r}=\sum_{n=0}^{\infty} B_{n, r}^{L}(x) \frac{t^{n}}{n !}
$$

It is well known that the complete Bell polynomials are defined by

$$
\exp \left(\sum_{j=1}^{\infty} x_{j} \frac{t^{j}}{j !}\right)=\sum_{n=0}^{\infty} B_{n}\left(x_{1}, x_{2}, \ldots, x_{n}\right) \frac{t^{n}}{n !} \quad(\text { see }[2-4,6,11,14])
$$

Then it can be shown that the complete Bell polynomials are given by

$$
B_{n}\left(x_{1}, x_{2}, \ldots, x_{n}\right)=\sum_{j_{1}+2 j_{2}+\cdots+n j_{n}=n} \frac{n !}{j_{1} ! j_{2} ! \cdots j_{n} !}\left(\frac{x_{1}}{1}\right)^{j_{1}}\left(\frac{x_{2}}{2 !}\right)^{j_{2}} \cdots\left(\frac{x_{n}}{n !}\right)^{j_{n}}
$$


where the sum runs over all nonnegative integers $j_{1}, j_{2}, \ldots, j_{n}$ satisfying $j_{1}+2 j_{2}+\cdots+n j_{n}=n$. The incomplete Bell polynomials are given by

$$
\frac{1}{k !}\left(\sum_{m=1}^{\infty} x_{m} \frac{t^{m}}{m !}\right)^{k}=\sum_{n=k}^{\infty} B_{n, k}\left(x_{1}, x_{2}, \ldots, x_{n-k+1}\right) \frac{t^{n}}{n !} \quad(n \geq 0)(\text { see }[6,11,14]) .
$$

Thus

$$
\begin{aligned}
& B_{n, k}\left(x_{1}, x_{2}, \ldots, x_{n-k+1}\right) \\
& \quad=\sum_{\pi(n, k)} \frac{n !}{j_{1} ! j_{2} ! \cdots j_{n-k+1} !}\left(\frac{x_{1}}{1 !}\right)^{j_{1}}\left(\frac{x_{2}}{2 !}\right)^{j_{2}} \cdots\left(\frac{x_{n-k+1}}{(n-k+1) !}\right)^{j_{n-k+1}},
\end{aligned}
$$

where the sum runs over the set $\pi(n, k)$ of all nonnegative integers $\left(j_{i}\right)_{i \geq 1}$ satisfying $j_{1}+j_{2}+$ $\cdots+j_{n-k+1}=k$ and $1 j_{1}+2 j_{2}+\cdots+(n-k+1) j_{n-k+1}=n$.

The complete and incomplete Bell polynomials are related by

$$
B_{n}\left(x_{1}, x_{2}, \ldots, x_{n}\right)=\sum_{k=1}^{n} B_{n, k}\left(x_{1}, x_{2}, \ldots, x_{n-k+1}\right) \quad(n \geq 1) .
$$

Let $f$ be a $C^{\infty}$-function, that is, $f$ is a function that has continuous derivatives of all orders on $(-\infty, \infty)$. Then by (8) we have

$$
\begin{aligned}
e^{f(x+t)} & =\exp \left(\sum_{j=0}^{\infty} f^{(j)}(x) \frac{t^{j}}{j !}\right) \\
& =\exp \left(f(x)+\sum_{j=1}^{\infty} f^{(j)}(x) \frac{t^{j}}{j !}\right) \\
& =e^{f(x)}\left(1+\sum_{n=1}^{\infty} B_{n}\left(f^{(1)}(x), f^{(2)}(x), \ldots, f^{(n)}(x)\right) \frac{t^{n}}{n !}\right),
\end{aligned}
$$

where $f^{(j)}(x)$ is the $j$ th derivative of $f(x)$, and $\exp (t)=e^{t}$.

We observe that

$$
\begin{aligned}
\frac{d^{m}}{d x^{m}} e^{f(x)} & =\left.\frac{\partial^{m}}{\partial x^{m}} e^{f(x+t)}\right|_{t=0}=\left.\frac{\partial^{m}}{\partial t^{m}} e^{f(x+t)}\right|_{t=0} \\
& =e^{f(x)} B_{m}\left(f^{(1)}(x), f^{(2)}(x), \ldots, f^{(m)}(x)\right) .
\end{aligned}
$$

From (12) and (13) we obtain the Kölbig-Coeffey equation

$$
\left.\frac{d^{m}}{d x^{m}} e^{f(x)}=e^{f(x)} B_{m}\left(f^{(1)}(x), f^{(2)}(x), \ldots, f^{(m)}(x)\right) \quad(m \geq 1) \text { (see }[5,12]\right) .
$$

The exponential incomplete $r$-Bell polynomials are defined by the generating function

$$
\frac{1}{k !}\left(\sum_{j=1}^{\infty} a_{j} \frac{t^{j}}{j !}\right)^{k}\left(\sum_{i=0}^{\infty} b_{i+1} \frac{t^{i}}{i !}\right)^{r}=\sum_{n=k}^{\infty} B_{n+r, k+r}^{(r)}\left(a_{1}, a_{2}, \ldots: b_{1}, b_{2}, \ldots\right) \frac{t^{n}}{n !} .
$$


From (15) we note that

$$
\begin{aligned}
& B_{n+r, k+r}^{(r)}\left(a_{1}, a_{2}, \ldots: b_{1}, b_{2}, \ldots\right) \\
& =\sum_{\Lambda(n, k, r)}\left[\frac{n !}{k_{1} ! k_{2} ! k_{3} \ldots}\left(\frac{a_{1}}{1 !}\right)^{k_{1}}\left(\frac{a_{2}}{2 !}\right)^{k_{2}}\left(\frac{a_{3}}{3 !}\right)^{k_{3}} \ldots\right] \\
& \quad \times\left[\frac{r !}{r_{0} ! r_{1} ! r_{2} \ldots}\left(\frac{b_{1}}{0 !}\right)^{r_{0}}\left(\frac{b_{2}}{1 !}\right)^{r_{1}}\left(\frac{b_{3}}{2 !}\right)^{r_{2}} \ldots\right],
\end{aligned}
$$

where $\Lambda(n, k, r)$ denotes the set of all nonnegative integers $\left(k_{i}\right)_{i \geq 1}$ and $\left(r_{i}\right)_{i \geq 0}$ such that

$$
\sum_{i \geq 1} k_{i}=k, \quad \sum_{i \geq 0} r_{i}=r, \quad \text { and } \quad \sum_{i \geq 1} i\left(k_{i}+r_{i}\right)=n \quad(\text { see }[4,6,14]) .
$$

Let $\left(a_{i}\right)_{i \geq 1}$ and $\left(b_{i}\right)_{i \geq 1}$ are sequences of positive integers. Then the number $B_{n+r, k+r}^{(r)}\left(a_{1}, a_{2}\right.$, $\left.\ldots ; b_{1}, b_{2}, \ldots\right)$ counts the number of partitions of an $(n+r)$-set into $(k+r)$ blocks satisfying:

- The first $r$ elements belong to different blocks;

- Any block of size $i$ containing no elements from the first $r$ elements can be colored with $a_{i}$ colors;

- Any block of size $i$ containing one element from the first $r$ elements can be colored with $b_{i}$ colors.

The complete $r$-Bell polynomials are given by

$$
\exp \left(\sum_{i=1}^{\infty} a_{i} \frac{t^{i}}{i !}\right)\left(\sum_{j=0}^{\infty} b_{j+1} \frac{t^{j}}{j !}\right)^{r}=\sum_{n=0}^{\infty} B_{n}^{(r)}\left(a_{1}, a_{2}, \ldots ; b_{1}, b_{2}, \ldots\right) \frac{t^{n}}{n !}
$$

(see $[4,6,11,14])$.

By (16) and (17) we get

$$
B_{n}^{(r)}\left(a_{1}, a_{2}, \ldots: b_{1}, b_{2}, \ldots\right)=\sum_{k=0}^{n} B_{n+r, k+r}^{(r)}\left(a_{1}, a_{2}, \ldots: b_{1}, b_{2}, \ldots\right) \quad(\text { see }[6]) .
$$

The incomplete and complete Bell polynomials have applications to such diverse areas as combinatorics, probability, algebra, and analysis. The number of monomials appearing in the incomplete Bell polynomial $B_{n, k}\left(x_{1}, x_{2}, \ldots, x_{n-k+1}\right)$ is the number of partitioning $n$ into $k$ parts, and the coefficient of each monomial is the number of partitioning $n$ as the corresponding $k$ parts. Also, the incomplete Bell polynomials $B_{n, k}\left(x_{1}, x_{2}, \ldots, x_{n-k+1}\right)$ appear in the Faà di Bruno formula concerning higher-order derivatives of composite functions (see [6]). In addition, the incomplete Bell polynomials can be used in constructing sequences of binomial type (see [16]), and there are certain connections between incomplete Bell polynomials and combinatorial Hopf algebras such as the Hopf algebra of word symmetric functions, the Hopf algebra of symmetric functions, and the Fa di Bruno algebra (see [1]). The complete Bell polynomials $B_{n}\left(x_{1}, x_{2}, \ldots, x_{n}\right)$ have applications to probability theory (see $[6,12,18])$. Indeed, the $n$th moment $\mu_{n}=E\left[X^{n}\right]$ of the random variable $X$ is the $n$th complete Bell polynomial in the first $n$ cumulants $\mu_{n}=B_{n}\left(\kappa_{1}, \kappa_{2}, \ldots, \kappa_{n}\right)$. The reader can refer to the Ph.D. thesis of Port [18] for many applications to probability theory and combinatorics. Many special numbers, like Stirling numbers of both kinds, Lah numbers, 
and idempotent numbers, appear in many combinatorial and number-theoretic identities involving complete and incomplete Bell polynomials. We refer the reader to the Introduction in [11] for further details.

The incomplete Lah-Bell polynomials (see (22)) and the complete Lah-Bell polynomials (see (25)) are respectively multivariate versions of the unsigned Lah numbers and the Lah-Bell numbers. Note here that the incomplete Bell polynomials (see (10)) and the incomplete Lah-Bell polynomials are related as given in (23), whereas the complete Bell polynomials (see (8)) and the complete Lah-Bell polynomials are related as given in (26). The incomplete $r$-extended Lah-Bell polynomials (see (30)) and the complete $r$-extended Lah-Bell polynomials (see (32)) are respectively extended versions of the incomplete LahBell polynomials and the complete Lah-Bell polynomials. Further, they are respectively multivariate versions of the $r$-Lah numbers and the $r$-extended Lah-Bell numbers.

The aim of this paper is to introduce the incomplete $r$-extended Lah-Bell polynomials and the complete $r$-extended Lah-Bell polynomials and to investigate some properties and identities for these polynomials. From these investigations we obtain some expressions for the $r$-Lah numbers and the $r$-extended Lah-Bell numbers as finite sums.

\section{Complete and incomplete $r$-extended Lah-Bell polynomials}

Let $f(t)=\frac{t}{1-t}$. Then we have

$$
f^{(n)}(t)=\frac{d^{n}}{d t^{n}} f(t)=\frac{n !}{(1-t)^{n+1}} \quad(n \geq 1)
$$

By (14) we get

$$
\left.\frac{d^{n}}{d t^{n}} e^{\frac{t}{1-t}}\right|_{t=0}=B_{n}(1 !, 2 !, \ldots, n !)
$$

From (2) we note that

$$
\left.\frac{d^{n}}{d t^{n}} e^{\frac{t}{1-t}}\right|_{t=0}=\left.\frac{d^{n}}{d t^{n}} \sum_{k=0}^{\infty} B_{k}^{L} \frac{t^{k}}{k !}\right|_{t=0}=B_{n}^{L}
$$

Therefore by (20) and (21) we obtain the following theorem.

Theorem 1 For $n \geq 1$, we have

$$
B_{n}^{L}=B_{n}(1 !, 2 !, \ldots, n !)=\sum_{k_{1}+2 k_{2}+\cdots+n k_{n}=n} \frac{n !}{k_{1} ! k_{2} ! \cdots k_{n} !} .
$$

Let us consider the incomplete Lah-Bell polynomials given by

$$
\frac{1}{k !}\left(\sum_{m=1}^{\infty} x_{m} t^{m}\right)^{k}=\sum_{n=k}^{\infty} B_{n, k}^{L}\left(x_{1}, x_{2}, \ldots, x_{n-k+1}\right) \frac{t^{n}}{n !},
$$

where $n, k \geq 0$ with $n \geq k$.

Note hat $B_{n, k}^{L}(1,1, \ldots, 1)=L(n, k)(n \geq k \geq 0)$. 
Indeed, by (10) and (22) we get

$$
B_{n, k}^{L}\left(x_{1}, x_{2}, \ldots, x_{n-k+1}\right)=B_{n, k}\left(1 ! x_{1}, 2 ! x_{2}, \ldots,(n-k+1) ! x_{n-k+1}\right) .
$$

From (23) we note that

$$
\begin{aligned}
L(n, k) & =B_{n, k}^{L}(1,1, \ldots, 1)=B_{n, k}(1 !, 2 !, \ldots,(n-k+1) !) \\
& =\sum_{\substack{j_{1}+j_{2}+\cdots+j_{n-k+1}=k \\
j_{1}+2 j_{2}+\cdots+(n-k+1) j_{n-k+1}=n}} \frac{n !}{j_{1} ! j_{2} ! \cdots j_{n-k+1} !} .
\end{aligned}
$$

Therefore by (23) we obtain the following proposition.

Proposition 2 For $n, k \geq 0$ with $n \geq k$, we have

$$
B_{n, k}^{L}\left(x_{1}, x_{2}, \ldots, x_{n-k+1}\right)=B_{n, k}\left(1 ! x_{1}, 2 ! x_{2}, \ldots,(n-k+1) x_{n-k+1}\right) .
$$

In addition,

$$
L(n, k)=\sum_{\substack{j_{1}+j_{2}+\cdots+j_{n-k+1}=k \\ j_{1}+2 j_{2}+\cdots+(n-k+1) j_{n-k+1}=n}} \frac{n !}{j_{1} ! j_{2} ! \cdots j_{n-k+1} !}
$$

From (23) we note that

$$
\begin{aligned}
B_{n, k}^{L}\left(\alpha x_{1}, \alpha x_{2}, \ldots, \alpha x_{n-k+1}\right) & =B_{n, k}\left(\alpha \cdot 1 ! x_{1}, \alpha \cdot 2 ! x_{2}, \ldots, \alpha \cdot(n-k+1) ! x_{n-k+1}\right) \\
& =\alpha^{k} B_{n, k}\left(1 ! x_{1}, 2 ! x_{2}, \ldots,(n-k+1) ! x_{n-k+1}\right) \\
& =\alpha^{k} B_{n, k}^{L}\left(x_{1}, x_{2}, \ldots, x_{n-k+1}\right) .
\end{aligned}
$$

We now consider the complete Lah-Bell polynomials given by

$$
\exp \left(\sum_{i=1}^{\infty} x_{i} t^{i}\right)=\sum_{n=0}^{\infty} B_{n}^{L}\left(x_{1}, x_{2}, \ldots, x_{n}\right) \frac{t^{n}}{n !}
$$

By (25) we get

$$
\begin{aligned}
B_{n}^{L}\left(x_{1}, x_{2}, \ldots, x_{n}\right) & =B_{n}\left(1 ! x_{1}, 2 ! x_{2}, \ldots, n ! x_{n}\right) \\
& =\sum_{l_{1}+2 l_{2}+\cdots+n l_{n-1}=n} \frac{n !}{l_{1} ! l_{2} ! \cdots l_{n} !} x_{1}^{l_{1}} x_{2}^{l_{2}} \cdots x_{n}^{l_{n}} \quad(n \geq 0) .
\end{aligned}
$$

From (22) and (25) we note that

$$
\begin{aligned}
1+\sum_{n=1}^{\infty} B_{n}^{L}\left(x_{1}, x_{2}, \ldots, x_{n}\right) \frac{t^{n}}{n !} & =\exp \left(\sum_{i=1}^{\infty} x_{i} t^{i}\right) \\
& =1+\sum_{k=1}^{\infty} \frac{1}{k !}\left(\sum_{i=1}^{\infty} x_{i} t^{i}\right)^{k}
\end{aligned}
$$




$$
\begin{aligned}
& =1+\sum_{k=1}^{\infty} \sum_{n=k}^{\infty} B_{n}^{L}\left(x_{1}, x_{2}, \ldots, x_{n-k+1}\right) \frac{t^{n}}{n !} \\
& =1+\sum_{n=1}^{\infty}\left(\sum_{k=1}^{n} B_{n, k}^{L}\left(x_{1}, x_{2}, \ldots, x_{n-k+1}\right) \frac{t^{n}}{n !} .\right.
\end{aligned}
$$

Therefore by (25) and (27) we obtain the following theorem.

Theorem 3 For $n \geq 1$, we have

$$
\begin{aligned}
B_{n}^{L}\left(x_{1}, x_{2}, \ldots, x_{n}\right) & =\sum_{k=1}^{n} B_{n, k}^{L}\left(x_{1}, x_{2}, \ldots, x_{n-k+1}\right) \\
& =\sum_{k=1}^{n} B_{n, k}\left(1 ! x_{1}, 2 ! x_{2}, \ldots,(n-k+1) ! x_{n-k+1}\right) .
\end{aligned}
$$

In addition, for $n \geq 1$, we have

$$
B_{n}^{L}\left(x_{1}, x_{2}, \ldots, x_{n}\right)=\sum_{k=1}^{n} \sum_{\pi(n, k)} \frac{n !}{l_{1} ! l_{2} ! \cdots l_{n-k+1} !} x_{1}^{l_{1}} x_{2}^{l_{2}} \cdots x_{n-k+1}^{l_{n-k+1}},
$$

where $\pi(n, k)$ denotes the set of all nonnegative integers $\left(l_{i}\right)_{i \geq 1}$ such that $l_{1}+l_{2}+\cdots+l_{n-k+1}=$ $k$ and $1 \cdot l_{1}+2 \cdot l_{2}+\cdots+(n-k+1) l_{n-k+1}=n$.

By (25) we easily get

$$
\sum_{n=0}^{\infty} B_{n}^{L}(1,1, \ldots, 1) \frac{t^{n}}{n !}=\exp \left(\sum_{i=1}^{\infty} t^{i}\right)=\exp \left(\frac{t}{1-t}\right)=\sum_{n=0}^{\infty} B_{n}^{L} \frac{t^{n}}{n !}
$$

From (28) we note that

$$
B_{n}^{L}(1,1, \ldots, 1)=B_{n}^{L} \quad(n \geq 0) .
$$

By Proposition 2, (24), and Theorem 3 we get

$$
\begin{aligned}
B_{n}^{L}(x, x, \ldots, x) & =\sum_{k=0}^{n} B_{n, k}^{L}(x, x, \ldots, x)=\sum_{k=0}^{n} B_{n, k}\left(1 ! x, 2 ! x, \ldots,(n-k+1) ! x_{n-k+1}\right) \\
& =\sum_{k=0}^{n} x^{k} B_{n, k}(1 !, 2 !, \ldots,(n-k+1) !)=\sum_{k=0}^{n} x^{k} L(n, k)=B_{n}^{L}(x) .
\end{aligned}
$$

Assume that $\left\{a_{i}\right\}_{i \geq 1}$ and $\left\{b_{i}\right\}_{i \geq 1}$ are sequences of positive integers. We define the incomplete r-extended Lah-Bell polynomials by

$$
\frac{1}{k !}\left(\sum_{j=1}^{\infty} a_{j} t^{j}\right)^{k}\left(\sum_{i=0}^{\infty} b_{i+1} t^{i}\right)^{2 r}=\sum_{n=k}^{\infty} B_{n+2 r, k+2 r}^{L}\left(a_{1}, a_{2}, \ldots: b_{1}, b_{2}, \ldots\right) \frac{t^{n}}{n !}
$$

where $k, r$ are nonnegative integers. 
From (30) we have

$$
\begin{aligned}
& B_{n+2 r, k+2 r}^{L}\left(a_{1}, a_{2}, \ldots: b_{1}, b_{2}, \ldots\right) \\
& \quad=B_{n+2 r, k+2 r}^{(2 r)}\left(1 ! a_{1}, 2 ! a_{2}, \ldots: 0 ! b_{1}, 1 ! b_{2}, \ldots\right) \\
& \quad=\sum_{\Lambda(n, k, 2 r)}\left[\frac{n !}{k_{1} ! k_{2} ! k_{3} ! \cdots} a_{1}^{k_{1}} a_{2}^{k_{2}} a_{3}^{k_{3}} \ldots\right]\left[\frac{(2 r) !}{r_{0} ! r_{1} ! r_{2} \ldots} b_{1}^{r_{0}} b_{2}^{r_{1}} b_{3}^{r_{2}} \ldots\right],
\end{aligned}
$$

where $\Lambda(n, k, 2 r)$ denotes the set of all nonnegative integers $\left\{k_{i}\right\}_{i \geq 1}$ and $\left\{r_{i}\right\}_{i \geq 0}$ such that $\sum_{i \geq 1} k_{i}=k, \sum_{i \geq 0} r_{i}=2 r$, and $\sum_{i \geq 1} i\left(k_{i}+r_{i}\right)=n$.

We define the complete r-extended Lah-Bell polynomials $B_{n}^{(L, 2 r)}\left(x \mid a_{1}, a_{2}, \ldots: b_{1}, b_{2}, \ldots\right)$ $(n \geq 0)$, which are given by

$$
\exp \left(x \sum_{j=1}^{\infty} a_{j} t^{j}\right)\left(\sum_{i=0}^{\infty} b_{i+1} t^{i}\right)^{2 r}=\sum_{n=0}^{\infty} B_{n}^{(L, 2 r)}\left(x \mid a_{1}, a_{2}, \ldots: b_{1}, b_{2}, \ldots\right) \frac{t^{n}}{n !}
$$

Thus we note that

$$
\begin{aligned}
\exp & \left(x \sum_{j=1}^{\infty} a_{j} t^{j}\right)\left(\sum_{i=0}^{\infty} b_{i+1} t^{i}\right)^{2 r} \\
& =\sum_{k=0}^{\infty} \frac{x^{k}}{k !}\left(\sum_{j=1}^{\infty} a_{j} t^{j}\right)^{k}\left(\sum_{i=0}^{\infty} b_{i+1} t^{i}\right)^{2 r} \\
& =\sum_{k=0}^{\infty} x^{k} \sum_{n=k}^{\infty} B_{n+2 r, k+2 r}^{L}\left(a_{1}, a_{2}, \ldots: b_{1}, b_{2}, \ldots\right) \frac{t^{n}}{n !} \\
& =\sum_{n=0}^{\infty} \sum_{k=0}^{n} x^{k} B_{n+2 r, k+2 r}^{L}\left(a_{1}, a_{2}, \ldots: b_{1}, b_{2}, \ldots\right) \frac{t^{n}}{n !} .
\end{aligned}
$$

From (32) and (33) we have

$$
B_{n}^{(L, 2 r)}\left(x \mid a_{1}, a_{2}, \ldots: b_{1}, b_{2}, \ldots\right)=\sum_{k=0}^{n} x^{k} B_{n+2 r, k+2 r}^{L}\left(a_{1}, a_{2}, \ldots: b_{1}, b_{2}, \ldots\right) \quad(n \geq 0) .
$$

By (18), (31), (32), and (34) we have

$$
\begin{aligned}
B_{n}^{(2 r)} & \left(1 ! a_{1}, 2 ! a_{2}, \ldots: 0 ! b_{1}, 1 ! b_{2}, \ldots\right) \\
& =\sum_{k=0}^{n} B_{n+2 r, k+2 r}^{(2 r)}\left(1 ! a_{1}, 2 ! a_{2}, \ldots: 0 ! b_{1}, 1 ! b_{2}, \ldots\right) \\
& =\sum_{k=0}^{n} B_{n+2 r, k+2 r}^{L}\left(a_{1}, a_{2}, \ldots: b_{1}, b_{2}, \ldots\right)=B_{n}^{(L, 2 r)}\left(1 \mid a_{1}, a_{2}, \ldots: b_{1}, b_{2}, \ldots\right) .
\end{aligned}
$$

Therefore by (31) and (34) we obtain the following theorem. 
Theorem 4 For $n \geq 0$, we have

$$
\begin{aligned}
B_{n}^{(L, 2 r)}\left(x \mid a_{1}, a_{2}, \ldots: b_{1}, b_{2}, \ldots\right) & =\sum_{k=0}^{n} x^{k} B_{n+2 r, k+2 r}^{L}\left(a_{1}, a_{2}, \ldots: b_{1}, b_{2}, \ldots\right) \\
& =\sum_{k=0}^{n} x^{k} B_{n+2 r, k+2 r}^{(2 r)}\left(1 ! a_{1}, 2 ! a_{2}, \ldots: 0 ! b_{1}, 1 ! b_{2}, \ldots\right) .
\end{aligned}
$$

From (30) we note that

$$
\begin{aligned}
\sum_{n=k}^{\infty} B_{n+2 r, k+2 r}^{L}(1,1, \ldots ; 1,1, \ldots) \frac{t^{n}}{n !} & =\frac{1}{k !}\left(\sum_{j=1}^{\infty} t^{j}\right)^{k}\left(\sum_{i=0}^{\infty} t^{i}\right)^{2 r} \\
& =\frac{1}{k !}\left(\frac{t}{1-t}\right)^{k}\left(\frac{1}{1-t}\right)^{2 r}=\sum_{n=k}^{\infty} L_{r}(n, k) \frac{t^{n}}{n !} .
\end{aligned}
$$

By (32) and (36) we get

$$
\begin{aligned}
\sum_{n=0}^{\infty} B_{n}^{(L, 2 r)}(x \mid 1,1, \ldots: 1,1, \ldots) \frac{t^{n}}{n !} & =\exp \left(x \sum_{j=1}^{\infty} t^{j}\right)\left(\sum_{i=0}^{\infty} t^{i}\right)^{2 r} \\
& =e^{x\left(\frac{t}{1-t}\right)} \cdot\left(\frac{1}{1-t}\right)^{2 r}=\sum_{n=0}^{\infty}\left(\sum_{k=0}^{n} x^{k} L_{r}(n, k)\right) \frac{t^{n}}{n !} .
\end{aligned}
$$

Thus by (36) and (37) we have

$$
\begin{aligned}
B_{n}^{(L, 2 r)}(x \mid 1,1, \ldots: 1,1, \ldots) & =\sum_{k=0}^{n} x^{k} B_{n+2 r, k+2 r}^{L}(1,1, \ldots: 1,1, \ldots) \\
& =\sum_{k=0}^{n} x^{k} L_{r}(n, k) .
\end{aligned}
$$

Therefore we obtain the following theorem.

Theorem 5 For $n \geq k \geq 0$, we have

$$
B_{n+2 r, k+2 r}^{L}(1,1, \ldots: 1,1, \ldots)=L_{r}(n, k)
$$

and

$$
B_{n}^{(L, 2 r)}(x \mid 1,1, \ldots: 1,1, \ldots)=\sum_{k=0}^{n} B_{n+2 r, k+2 r}^{L}(1,1, \ldots: 1,1, \ldots)=\sum_{k=0}^{n} x^{k} L_{r}(n, k) .
$$

From (36) and (31) we note that

$$
\begin{aligned}
L_{r}(n, k) & =B_{n+2 r, k+2 r}^{L}(1,1, \ldots: 1,1, \ldots) \\
& =B_{n+2 r, k+2 r}^{(2 r)}(1 !, 2 !, \ldots: 1 !, 2 !, \ldots) \\
& =\sum_{\Lambda(n, k, 2 r)} \frac{n !}{k_{1} ! k_{2} ! \cdots} \frac{(2 r) !}{r_{0} ! r_{1} ! \cdots} .
\end{aligned}
$$


Corollary 6 For $n, k, r \geq 0$ with $n \geq k$, we have

$$
L_{r}(n, k)=\sum_{\Lambda(n, k, 2 r)} \frac{n !}{k_{1} ! k_{2} ! \cdots} \cdot \frac{(2 r) !}{r_{0} ! r_{1} ! \cdots}
$$

where $\Lambda(n, k, 2 r)$ denotes the set of all nonnegative integers $\left\{k_{i}\right\}_{i \geq 1}$ and $\left\{r_{i}\right\}_{i \geq}$ such that $\sum_{i \geq 1} k_{i}=k, \sum_{i \geq 0} r_{i}=2 r$, and $\sum_{i \geq 1} i\left(k_{i}+r_{i}\right)=n$.

Now we observe that

$$
\begin{aligned}
\exp \left(\sum_{i=1}^{\infty} x_{i} t^{i}\right) & =1+\sum_{k=1}^{\infty} \frac{1}{k !}\left(\sum_{i=1}^{\infty} x_{i} t^{i}\right)^{k} \\
& =1+\frac{1}{1 !} \sum_{i=1}^{\infty} x_{i} t^{i}+\frac{1}{2 !}\left(\sum_{i=1}^{\infty} x_{i} t^{i}\right)^{2}+\frac{1}{3 !}\left(\sum_{i=1}^{\infty} x_{i} t^{i}\right)^{3}+\cdots \\
& =\sum_{k=0}^{\infty} \sum_{m_{1}+2 m_{2}+\cdots+k m_{k}=k} \frac{1}{m_{1} ! m_{2} ! \cdots m_{k} !} x_{1}^{m_{1}} x_{2}^{m_{2}} \cdots x_{k}^{m_{k}} t^{k}
\end{aligned}
$$

and

$$
\left(\sum_{j=0}^{\infty} y_{j+1} t^{j}\right)^{2 r}=\sum_{m=0}^{\infty} \sum_{l_{1}+\cdots+l_{2 r}=m} y_{l_{1}+1} y_{l_{2}+1} \cdots y_{l_{2 r}+1} t^{m} .
$$

By (39) and (40) we get

$$
\begin{aligned}
& \exp \left(\sum_{i=1}^{\infty} x_{i} t^{i}\right)\left(\sum_{j=0}^{\infty} y_{j+1} \frac{t^{j}}{j !}\right)^{2 r} \\
& =\sum_{n=0}^{\infty} n !\left(\sum_{k=0}^{n} \sum_{m_{1}+2 m_{2}+\cdots+k m_{k}=k} \sum_{l_{1}+l_{2}+\cdots+l_{2 r}=n-k} \frac{1}{m_{1} ! m_{2} ! \cdots m_{k} !} x_{1}^{m_{1}} x_{2}^{m_{2}} \cdots x_{k}^{m_{k}}\right. \\
& \left.\quad \times y_{l_{1}+1} y_{l_{2}+1} \cdots y_{l_{2}+1}\right) \frac{t^{n}}{n !} .
\end{aligned}
$$

Therefore by (32) and (41) we obtain the following theorem.

Theorem 7 For $n, r \geq 0$, we have

$$
\begin{aligned}
& B_{n}^{(L, 2 r)}\left(1 \mid x_{1}, x_{2}, \ldots: y_{1}, y_{2}, \ldots\right) \\
& \quad=n ! \sum_{k=0}^{n} \sum_{m_{1}+2 m_{2}+\cdots+k m_{k}=k} \sum_{l_{1}+l_{2}+\cdots+l_{2 r}=n-k} \frac{1}{m_{1} ! m_{2} ! \cdots m_{k} !} x_{1}^{m_{1}} \cdots x_{k}^{m_{k}} y_{l_{1}+1} y_{l_{2}+1} \cdots y_{l_{2 r}+1} .
\end{aligned}
$$

Remark For $n \geq 0$, we have

$$
B_{n+2 r, k+2 r}^{L}(x, x, \ldots: 1,1, \ldots)=x^{k} B_{n+2 r, k+2 r}^{L}(1,1, \ldots: 1,1, \ldots) .
$$


Thus we note that

$$
\sum_{k=0}^{n} B_{n+2 r, k+2 r}^{L}(x, x, \ldots: 1,1, \ldots)=B_{n, r}^{L}(x) \quad(n \geq 0) .
$$

\section{Conclusion}

There are various methods of studying special numbers and polynomials, for example, generating functions, combinatorial methods, umbral calculus, $p$-adic analysis, differential equations, probability theory, orthogonal polynomials, and special functions. These ways of investigating special polynomials and numbers can be also applied to degenerate versions of such polynomials and numbers. Indeed, in recent years, many mathematicians have drawn their attention to studies of degenerate versions of many special polynomials and numbers by using the aforementioned means $([9,10,14]$ and references therein).

The incomplete and complete Bell polynomials arise in many different contexts as we stated in the Introduction. For instance, many special numbers, like Stirling numbers of both kinds, Lah numbers, and idempotent numbers, appear in many combinatorial and number-theoretic identities involving complete and incomplete Bell polynomials.

In this paper, we introduced the incomplete $r$-extended Lah-Bell polynomials and the complete $r$-extended Lah-Bell polynomials respectively as multivariate versions of $r$-Lah numbers and the $r$-extended Lah-Bell numbers and investigated some properties and identities for these polynomials. As corollaries of these results, we obtained some expressions for the $r$-Lah numbers and the $r$-extended Lah-Bell numbers as finite sums.

It would be very interesting to explore many applications of the incomplete and complete $r$-extended Lah-Bell polynomials as the incomplete and complete Bell polynomials have diverse applications.

\section{Acknowledgements}

The authors would like to thank the reviewers for their valuable comments and suggestions and Jangjeon Research Institute for Mathematical Sciences for the support of this research.

\section{Funding}

Not applicable.

Availability of data and materials

Not applicable.

Competing interests

The authors declare that they have no competing interests.

Authors' contributions

TK and DSK conceived of the framework and structured the whole paper; DSK and TK wrote the paper; LCJ, HL, and HYK checked the results of the paper; DSK and TK completed the revision of the paper. All authors have read and approved the final version of the manuscript.

\section{Author details}

'Department of Mathematics, Kwangwoon University, Seoul 139-701, Republic of Korea. ${ }^{2}$ Department of Mathematics, Sogang University, Seoul 121-742, Republic of Korea. ${ }^{3}$ Graduate School of Education, Konkuk University, Seoul 05029, Republic of Korea.

\section{Publisher's Note}

Springer Nature remains neutral with regard to jurisdictional claims in published maps and institutional affiliations. 


\section{References}

1. Aboud, A., Bultel, J.-P., Chouria, A., Luque, J.-G., Mallet, O.: Word Bell polynomials. Sémin. Lothar. Comb. 75, B75h (2017)

2. Abramowitz, M., Stegun, I.A.: Handbook of Mathematical Functions with Formulas, Graphs, and Mathematical Tables. National Bureau of Standards Applied Mathematics Series, vol. 55. U.S. Government Printing Office, Washington (1964). For sale by the Superintendent of Documents

3. Broder, A.Z: The $r$-Stirling numbers. Discrete Math. 49(3), 241-259 (1984)

4. Carlitz, L.: Arithmetic properties of the Bell polynomials. J. Math. Anal. Appl. 15, 33-52 (1966)

5. Coffey, M.W.: A set of identities for a class of alternating binomial sums arising in computing applications. arXiv:math-ph/0608049

6. Comtet, L.: Advanced Combinatorics. The Art of Finite and Infinite Expansions, revised and enlarged edn. Reidel, Dordrecht (1974)

7. Kim, D.S., Kim, T.: Lah-Bell numbers and polynomials. Proc. Jangjeon Math. Soc. 23(4), 577-586 (2020)

8. Kim, D.S., Kim, T.: $r$-Extended Lah-Bell numbers and polynomials associated with $r$-Lah numbers. Proc. Jangjeon Math. Soc. 24(1), 1-10 (2021). arXiv:2008.06155

9. Kim, H.K.: Degenerate Lah-Bell polynomials arising from degenerate Sheffer sequences. Adv. Differ. Equ. 2020, Article ID 687 (2020)

10. Kim, H.K., Lee, D.S.: Note on extended Lah-Bell polynomials and degenerate extended Lah-Bell polynomials. Adv. Stud. Contemp. Math. (Kyungshang) 30(4), 547-558 (2020)

11. Kim, T., Kim, D.S., Jang, G.-W.: On central complete and incomplete Bell polynomials I. Symmetry 11(2), Article ID 288 (2019)

12. Kölbig, K.S.: The complete Bell polynomials for certain arguments in terms of Stirling numbers of the first kind. J. Comput. Appl. Math. 51, 113-116 (1994)

13. Kucukoglu, I., Simsek, Y.: Observations on identities and relations for interpolation functions and special numbers. Adv. Stud. Contemp. Math. (Kyungshang) 28(1), 41-56 (2018)

14. Kwon, J., Kim, T., Kim, D.S., Kim, H.Y.: Some identities for degenerate complete and incomplete $r$-Bell polynomials. J. Inequal. Appl. 2020, Article ID 23 (2020)

15. Ma, Y., Kim, D.S., Kim, T., Kim, H., Lee, H.: Some identities of Lah-Bell polynomials. Adv. Differ. Equ. 2020, Article ID 510 (2020)

16. Mihoubi, M.: Bell polynomials and binomial type sequences. Discrete Math. 308(12), 2450-2459 (2008)

17. Nyul, G., Racz, G.: The $r$-Lah numbers. Discrete Math. 338(10), 1660-1666 (2015)

18. Port, D.: Polynomial maps with applications to combinatorics and probability theory. Ph.D. thesis, Massachusetts Institute of Technology (1994)

19. Roman, S.: The Umbral Calculus. Pure and Applied Mathematics, vol. 111. Academic Press, New York (1984)

\section{Submit your manuscript to a SpringerOpen ${ }^{\circ}$ journal and benefit from:}

- Convenient online submission

- Rigorous peer review

- Open access: articles freely available online

- High visibility within the field

- Retaining the copyright to your article

Submit your next manuscript at $\boldsymbol{~ s p r i n g e r o p e n . c o m ~}$ 